\title{
An IoT and AI based Flood Monitoring and Rescue System
}

\author{
Mr. Ajim I. Pathan \\ Department of Computer Science and Engineering \\ Walchand College of Engineering, Sangli \\ Sangli,India \\ Ms. Nikita L. Gaikwad \\ Department of Computer Science and Engineering \\ Walchand College of Engineering, Sangli \\ Sangli,India
}

\author{
Ms. Aishwarya P. Kulkarni \\ Department of Computer Science and Engineering \\ Walchand College of Engineering, Sangli \\ Sangli,India \\ Ms. Prajakta M. Powar \\ Department of Computer Science and Engineering \\ Walchand College of Engineering, Sangli \\ Sangli,India
}

\author{
Prof. Anil R. Surve \\ Department of Computer Science and Engineering \\ Walchand College of Engineering, Sangli \\ Sangli,India
}

\begin{abstract}
One of the biggest natural disasters India had ever faced was floods in Western Maharashtra in August 2005 and August 2019. The primary objective of this research is to provide an innovative and sustainable solution required to monitor flood situations and perform rescue operations in the worst affected Sangli, Kolhapur and Satara districts. This attempt aims at providing IoT-based flood detection and AI-based rescue operation through the system. This research makes use of Internet of Things and Artificial Intelligence technologies to provide a reliable and implementable solution for the above problem. This innovative approach uses Arduino Uno microcontroller for flood detection based on rise of water level, humidity, atmospheric pressure and temperature measured through BMP180 sensor. Raspberry $\mathrm{Pi}$ has been used for carrying out drone-based rescue operations by detecting the number of people stuck in flooded regions using YOLO object detection algorithm and notifying concerned authorities about them through web portal and mobile application.
\end{abstract}

Keywords - Artificial Intelligence, Drones, Flood Monitoring System, Flood Rescue System, Internet of Things

\section{INTRODUCTION}

The world's climate is drastically changing because of natural calamities like hurricanes, tornadoes, volcanic eruptions, earthquakes, tsunamis, storms, floods and which is only the outcome of human activities such as pollution, cutting trees and many more hazardous activities. These natural disasters cause great damage and destroy the smooth living of human life causing the reconstruction of all things including the most important basic needs: food, shelter, clothing and water.

The Flood is one of the most frequent natural calamities India is ever facing. In August 2005 [9], due to heavy rainfalls, the western Maharashtra faced severe problems specifically in Sangli, Kolhapur and Satara districts and impacted livelihood of people, housing and farmlands. Kolhapur and Sangli had received heavy incessant rainfall and had broken the record of many previous years. Many of them evacuated to safer places but because of lack of management, precautions and technological advancement, flooding caused loss of human life, livestock, destruction of plants, property and deterioration of health conditions owing to waterborne diseases. Recently in 2019 there was heavy rainfall more than 2005, breaking the one-month record in just 9-10 days and the water level was above the danger line of Panchganga and Krishna river. The heavy incessant rainfalls in Radhanagari, Kalammawadi dam area, rising of urban areas near river basins in Kolhapur and Sangli area, backwater of Almatti dam causes the swelling of Krishna and Panchganga river and so the disaster has happened and many inhabitants near the river banks of Krishna, Panchganga and Koyana have been displaced. People faced a lot of hardship to evacuate to safer places. Hence, it is important to design a flood control mechanism. The pervasive computing technology is useful in such areas, which utilizes context to deal with relevant information and services in an effective manner [6].

This paper proposes the IoT and AI based flood monitoring and rescue system which is divided in two parts. The IoT-based part is for prevention of flood which detects the rise in water level and triggers alarm for alerting nearby people. The AIbased rescue part detects the number of people stuck in flooded regions and notifies concerned authorities through web portal and mobile application.

Section II explains previous research in related fields. Section III explains the methodology. Section IV analyzes the 
result of the experiment. Section $\mathrm{V}$ concludes the paper with an outline of contribution and the future work.

\section{LITERATURE REVIEW}

Numerous literatures related to IoT-based sensors and Computer Vision for flood monitoring and mapping were outlined [1] by Arshad B.; Ogie R.; Barthelemy J.; Pradhan B.; Verstaevel N. and Perez P. This attempt reviewed different applications aided with IoT and Computer Vision for better monitoring and mapping of floods.

Wahidah Md. Shah, F. Arif, A. A. Shahrin and Aslinda Hassan presented a flood warning system [2] based on IoT that was able to detect water level and calculate the speed of water level increased and alert nearby residents. The experiments were conducted in a controlled environment for testing the implemented system.

Pan, J.; Yin, Y; Xiong, J.; Luo, W.; Gui, G. and Sari, H. developed a network of automated surveillance composed of distant measuring stations and a control center [3]. They conducted tests using three methods, including the method of difference, dictionary learning and deep learning.

Elena Ridolfi and Piergiorgio Manciola proposed water level observations from drones at a dam site [4] which makes use of a sensing device composed of a drone and a camera to assess the water level.

A general-purpose design for context-consciously dependent flood control system with regard to contextconsciousness was demonstrated by Widiasari I, Nugro $\mathrm{L}$ and Widyawan in the form of WSN-based flood detection and monitoring device model [5].

Reference [7] shows how drones can be used during the times of natural disasters like Kerala floods in India. Since the ban imposed on flying drones in India had been lifted [8], they can be very effective during flood rescue operations.

As per reference [10], drones can also be used to deliver critical humanitarian aid. Customized drones can be built for carrying out rescue and relief operations in a better manner [11] [12].

\section{METHODOLOGY}

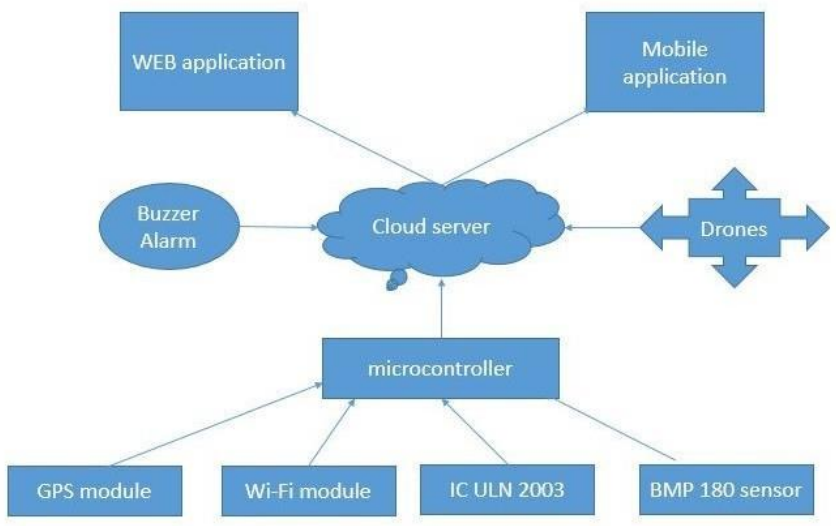

Fig. 1. System Architecture

As shown in Fig.1, All the data from the sensors are collected by microcontroller Arduino Uno. NODEMCUESP8266 WIFI development board is used to upload data from Arduino Uno to the Amazon Web Services cloud platform where data processing and analytics will be generated. Data about atmospheric pressure, water level height, humidity and temperature is collected using only one sensor called pressure sensor (BMP180 Barometric). IC ULN 2003 is used to show water level by blinking LED at each level. Data made available on a single website and mobile application is accessible to the concerned authorities and subscribed users for flood monitoring and decision-making. People who subscribe to this system can also receive alerts and notifications regarding water level in certain areas.

Drones can be used by integrating Raspberry pi as a rescue mechanism that will improve the quality of rescue operations and help in finding people stuck in flood and providing relief resources to needy people. With the help of camera attached to drones, pictures of flood affected areas are captured and those images are processed using YOLOv3 (You Only Look Once) state-of-the-art deep learning based object detection algorithm of Computer Vision library named ImageAI to find the count of people stuck in flood. It performs object detection on any image using pre-trained models trained on $\mathrm{COCO}$ dataset. Using its ObjectDetection class allows us to detect and recognize 80 possible objects. To make it simple, this sytem is only concerned about detecting number of people, ignoring other objects. This mechanism can help the government in rescue operations and to provide required resources to needy people.

NEO6M GPS Module is used to get the latitude and longitude of the location wherever help is required. The data is uploaded to AWS cloud with the help of NODEMCU ESP8266 WIFI development board. Alerts and notifications about the water level in certain areas are also sent to the subscribed users.

The web portal and mobile application give information about safe places and flood affected places, so people can move to safe places. Also, there is information about available shelters for flood victims. This system also provides facility for donations for flood victims through the web portal. Information about all volunteers for each area and various government schema is made available.

\section{RESULTS}

Following results are obtained through this research at Walchand College of Engineering, Sangli:

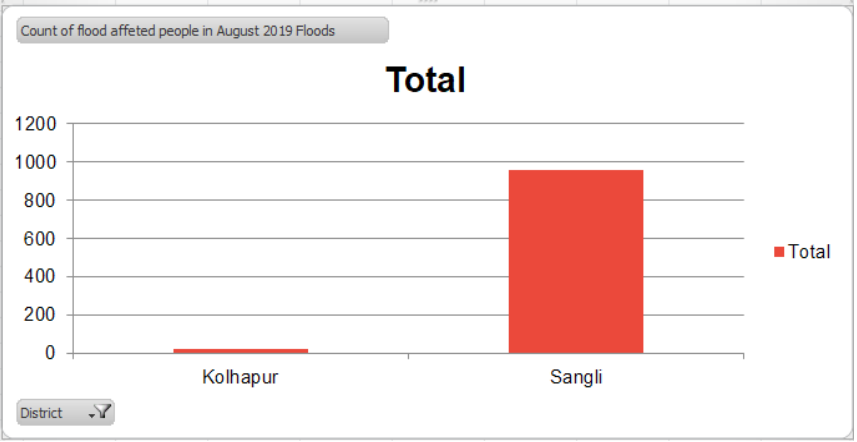

Fig. 2. Total number of August 2019 Flood victims in Sangli and Kolhapur districts

Fig. 2 visualizes data of 1000 people collected from collector office of Sangli that shows total number of people affected in 
August 2019 Floods in Sangli and Kolhaur districts. The Xaxis shows districts whereas $\mathrm{Y}$-axis represents number of people.

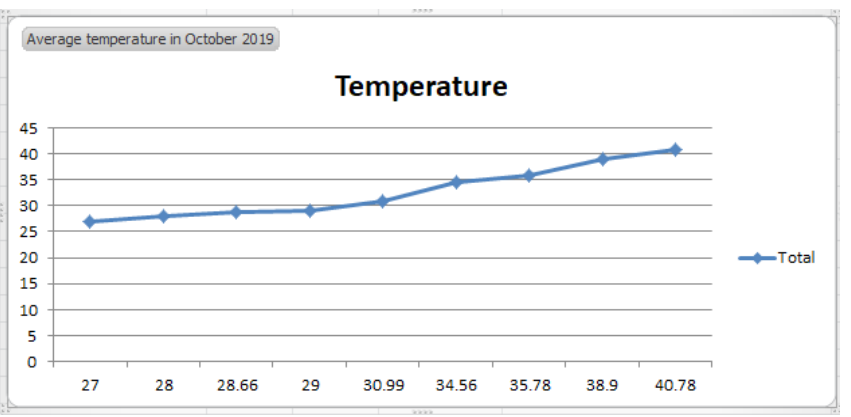

Fig. 3. Visualization of average temperature observed in September 2019

Fig. 3. shows average temperature observed in September 2019 at Sangli. Total number of observations and average temperature (in Celsius) for those observations are represented on $\mathrm{Y}$-axis and $\mathrm{X}$-axis respectively.

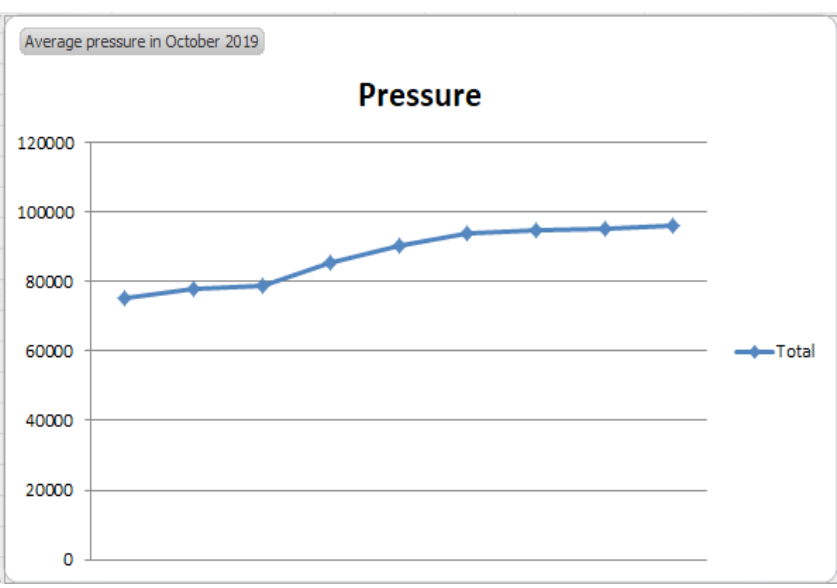

Fig. 4. Visualization of average atmospheric pressure observed in September 2019

The average atmospheric pressure in September 2019 at Sangli can be seen in Fig. 4. The values of shown observations are recorded through the system and are in Pascal unit.

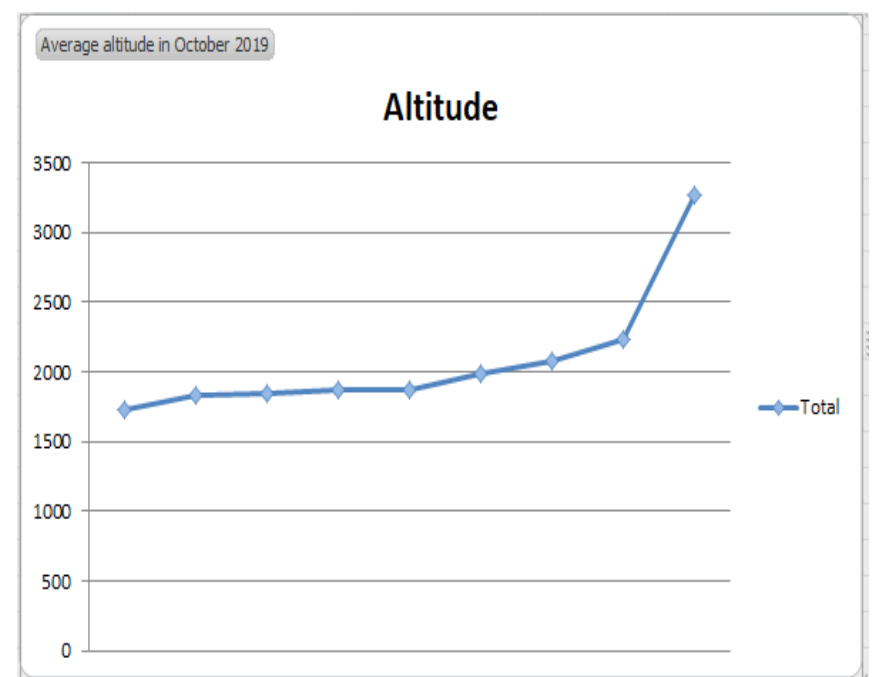

Fig. 5. Visualization of average altitude observed in September 2019
Fig. 5. depicts average altitude observed in September 2019 at Sangli. This helps to understand the water level heights represented on $\mathrm{Y}$-axis and $\mathrm{X}$-axis.

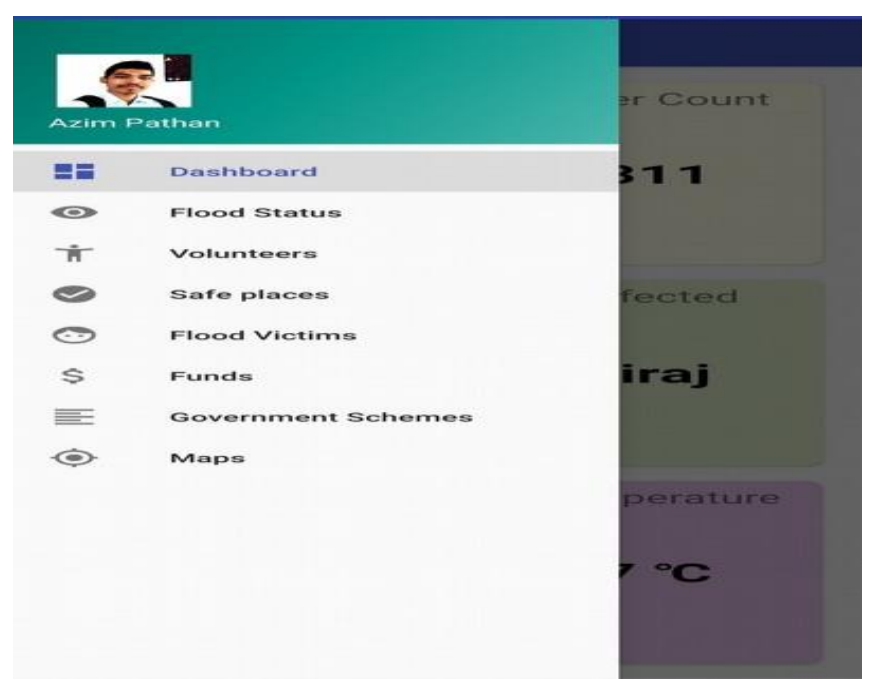

Fig. 6. Android Application

The main screen of android application is shown in Fig. 6. Options such as Maps, Governments schemes and Notices, Funds collected, etc are available for user.

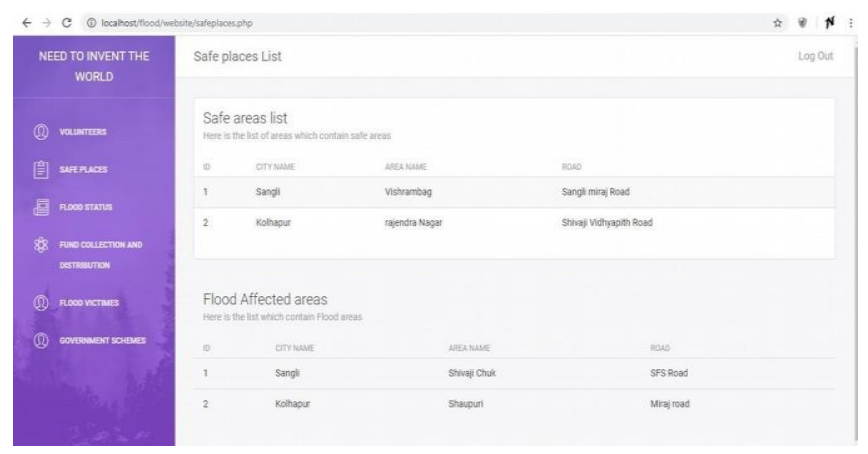

Fig. 7. Web portal

Fig. 7. shows web portal where users are able to see volunteers list, safe places list, flood status, fund collection and distribution, flood victims and government schemes.

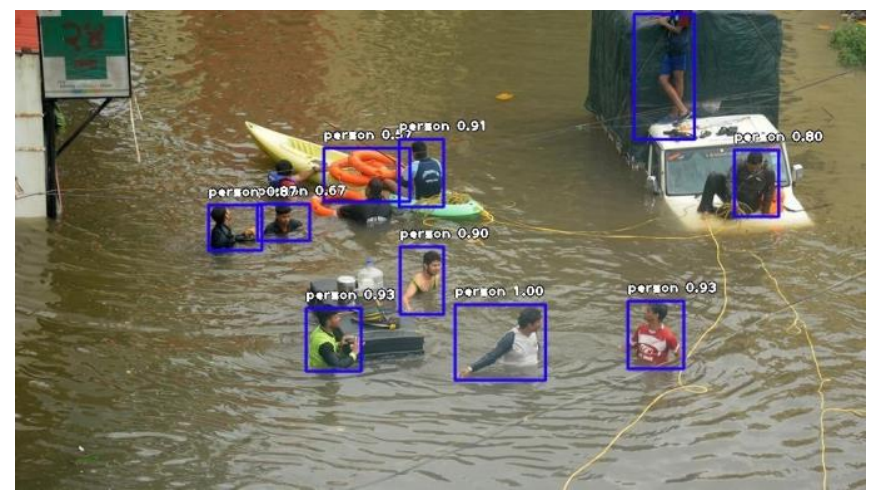

Fig. 8. Test image for counting people stuck in flood As shown in Fig. 8, 10 out of 11 people have been detected correctly that can be very helpful in rescue operations for finding how many people are stuck in flood at particular location. 


\section{CONCLUSION}

The IoT-based early detection of the flood helps to save many lives and enables real-time updates about water level to nearby people so they can relocate to safe places with their valuables. The detection helps to prevent damage of houses and businesses due to floods. This innovative approach assists concerned authorities to have access to real-time data in order to generate reports and perform analysis. express gratitude to Walchand College of Engineering, Sangli for providing opportunity for conducting this research.

\section{ACKNOWLEDGMENT}

We express gratitude to Walchand College of Engineering, Sangli for providing an opportunity for conducting this research.

\section{REFERENCES}

[1] Bilal Arshad, Robert Ogie, Johan Barthelemy, Biswajeet Pradhan, Nicolas Verstaevel and Pascal Perez, "Computer Vision and IoTBased Sensors in Flood Monitoring and Mapping: A Systematic Review", Nov 16,2019

[2] Wahidah Md. Shah, F. Arif, A. A. Shahrin and Aslinda Hassan, "The Implementation of an IoT-Based Flood Alert System", International Journal of Advanced Computer Science and Applications(IJACSA), Volume 9 Issue 11, 2018

[3] Pan, J.; Yin, Y; Xiong, J.; Luo, W.; Gui, G. and Sari, H., "Deep Learning-Based Unmanned Surveillance Systems for Observing Water Levels", IEEE Access, Volume 6, Nov 28, 2018

[4] Ridolfi, E. and Manciola, P., "Water Level Measurements from Drones: A Pilot Case Study at a Dam Site", Water 2018, Volume 10, Issue 3, Mar 9, 2018
[5] Indrastanti Widiasari, Lukito Nugroho, Widyawan Widyawan, A general purpose model for context aware based flood monitoring system, Jurnal Teknologi, June, 2016

[6] Anil Surve, Vijay Ghorpade, Pervasive Context-Aware Computing Survey of Context-aware ubiquitious middleware systems, Internationa Journal of Engineering Research and Technology, ISSN 0974-3154, Volume 10, Number 1, 2017

[7] Prince Diwaker, Kerala floods and drones, Geospatial World Forum, Aug 22, 2018. Accessed on: Aug 30, 2019. [Online]. Available: https://www.geospatialworld.net/blogs/kerala-floods-and-drones/

[8] Lysander Fernandes, Flying drones is now legal in India, here's all you need to know, The News Minute, Dec 02, 2018. Accessed on: Sept 15, 2019. [Online]. Available: https://www.thenewsminute.com/article/flying-drones-now-legalindiaheres-all-you-need-know-92558

[9] Shoumojit Banerjee, Kolhapur, Sangli face renewed flood threat as heavy rain batters western Maharashtra, The Hindu, Sept 10, 2019. Accessed on: Sept 16, 2019. [Online]. Available: https://www.thehindu.com/news/states/kolhapur-sangli-facerenewedflood-threat-as-heavy-rain-batters-western-maharashtra/ article29378391.ece

[10] Sam Sherman, Using drones to deliver critical humanitarian aid, Medium, Jun 7, 2018. Accessed on: Oct 3, 2019. [Online]. Available: https://medium.com/frontier-technology-livestreaming/using-dronestodeliver-critical-humanitarian-aid-1b578253fb76

[11] "HOW TO BUILD YOUR OWN DRONE FOR \$99”, May 6, 2018 Accessed on: Oct 10, 2019. [Online]. Available: https://thedronegirl.com/2018/05/06/build-your-own-drone/

[12] Jack Brown, HOW TO BUILD A DRONE: CONSTRUCT YOUR DRONE FROM SCRATCH. Accessed on: Oct 14, 2019. [Online]. Available: https://www.mydronelab.com/blog/how-to-build-adrone.html 